\title{
Development of in vivo Plant Propagation Protocol in Euphorbia milii var. 'Pink Bold Beauty'
}

\author{
D.B. Kapadiya*, Alka Singh, A.J. Bhandari, A.I. Patel and Krishna Patel \\ Department of Floriculture and Landscape Architecture, ASPEE College of Horticulture and \\ Forestry, Navsari Agricultural University, Navsari - 396 450, Gujarat, India \\ *Corresponding author
}

\section{A B S T R A C T}

\section{Keywords}

Euphorbia milii, in vivo propagation, Plant growth retardants, IBA, Rooting media, Cuttings.

\section{Article Info}

Accepted: 04 October 2017 Available Online: 10 December 2017
Experiment was conducted to develop plant propagation protocol via cutting in Euphorbia milii with the basic objective to enhance branching to obtain more number of cuttings for rooting and to standardize rooting treatment and rooting media. Pinching and foliar application of plant growth retardants significantly influenced vegetative growth and branching pattern of Euphorbia milii. Reduced plant height with thickest stem and maximum branches with more number of leaves was observed in pinched plants treated with Alar at $300 \mathrm{mg} / \mathrm{l}\left(\mathrm{P}_{1} \mathrm{R}_{6}\right)$ in var. 'Pink Bold Beauty'. Further, Maximum number of cuttings $(7.19,7.47)$ for propagation was obtained from pinched plants with foliar application of Alar at $300 \mathrm{mg} / \mathrm{l}$ during the experiment. Treatment of IBA $(0,250,500,750$ $\mathrm{mg} / \mathrm{l}$ ) and rooting media (sand, cocopeat and cocopeat + banana pseudo stem fibers) significantly influenced rooting in cuttings of Euphorbia milii and their shoot growth. Cuttings treated with IBA $(750 \mathrm{mg} / \mathrm{l})$ as quick deep method and stumped in cocopeat media $\left(\mathrm{M}_{2} \mathrm{I}_{4}\right)$ showed early sprouting with highest sprouting percentage. Cuttings of Euphorbia planted in cocopeat media after treatment of $750 \mathrm{mg} / \mathrm{l} \mathrm{IBA}\left(\mathrm{M}_{2} \mathrm{I}_{4}\right)$ showed higher number of roots per cutting $(14.37,15.71)$, maximum root length $(7.98,9.27)$ and higher survival percentage $(91.52,89.03 \%)$ in var. 'Pink Bold Beauty' during the consecutive years of study.

\section{Introduction}

Euphorbia milii (Crown of thorns, Christ plant and Christ-thorn) is a succulent species of flowering plant in the spurge family Euphorbiaceae and native to Madagascar. The species is mainly appreciated for the beauty of the inflorescence, continuous flowering and the hardiness of the plants (Jankalski, 2000). The plants of Euphorbia can be grown yearround in dry, high temperature and high solar radiation areas as potted, bedding, or garden plants (Jankalski, 2000). Euphorbia milii is a much esteemed plant for pot culture owing to its brilliant inflorescences. Crown of thorns is commercially propagated by tip cuttings (Smoley, 2000), but slow-growing and limited-branching habit is a major hurdle for propagation.

Physical control of apical dominance thorough pinching and application of plant growth retardants viz. Alar and TIBA aids to improve branching pattern in plants which can help for rapid multiplication through cuttings as quality planting material. Further, 
rooting media and IBA treatment promotes rooting of ornamental plant cuttings. Hence, an experiment was planned in two parts to develop in vivo plant propagation protocol in Euphorbia milii in var. 'Pink Bold Beauty' with following objectives. Part -1 was framed to study the effect of pinching and different plant growth retardants on branching habit of Euphorbia plants to obtain quality planting material for rapid multiplication. Part - 2 was designed for the standardization of rooting media and IBA concentration for efficient rooting in Euphorbia cuttings.

\section{Materials and Methods}

The present study was conducted in two different parts under naturally ventilated polyhouse located at ATC of Soilless System, Dept. of Floriculture and Landscape Architecture, ACHF, NAU, Navsari, Gujarat during 2015-2017. Both the experiments were laid out in Completely Randomized Design with Factorial concept (FCRD) and repeated thrice.

In part - 1, factor one consisted of two levels one being with pinching $\left(\mathrm{P}_{1}\right)$ and another without pinching $\left(\mathrm{P}_{2}\right)$ and factor two consists of 7 levels of foliar sprays, $50 \mathrm{mg} / \mathrm{l}$ TIBA $\left(\mathrm{R}_{1}\right), 100 \mathrm{mg} / \mathrm{l}$ TIBA $\left(\mathrm{R}_{2}\right), 150 \mathrm{mg} / \mathrm{l}$ TIBA $\left(\mathrm{R}_{3}\right), 100 \mathrm{mg} / \mathrm{l}$ Alar $\left(\mathrm{R}_{4}\right), 200 \mathrm{mg} / \mathrm{l}$ Alar $\left(\mathrm{R}_{5}\right)$, $300 \mathrm{mg} / \mathrm{l}$ Alar $\left(\mathrm{R}_{6}\right)$ and control i.e. no spray $\left(\mathrm{R}_{7}\right)$.

Pinching was performed at 5-6 $\mathrm{cm}$ height after transplanting of plants in pots. Apical portion of shoot was cut with the help of sharp secateurs. Spraying was followed two days after pinching at 15 days interval twice. The data on plant height $(\mathrm{cm})$, stem girth $(\mathrm{cm})$, number of leaves per plant, number of branches per plant were recorded at 30 and 60 DAS and number of cuttings suitable for rooting from each plant were harvested and recorded during the experiment.
In case of part -2 , uniform semi hardwood cuttings were collected from part -1 and kept for overnight to prevent latex accumulation at basal end. Next day basal end of cuttings were treated with $0,250,500$ and $750 \mathrm{mg} / \mathrm{l}$ Indole3-Butyric Acid (IBA) by quick deep method. Cuttings were placed into trays filled with three different subtracts [sand (1 part), cocopeat (1 part) and cocopeat + banana pseudostem fiber $(3: 1 \mathrm{v} / \mathrm{v})]$.

Rooting media were drenched every 15 days with $2 \mathrm{~g} /$ /itre copper oxychloride for fungal disease control. Data on the sprouting percentage, days taken for new sprouting, length of shoot number of roots per cutting, root length and survival percentage were collected at 60 days after planting.

The statistical analysis was done by adopting the appropriate standard error (S.Em \pm ) method in each case as suggested by Panse and Sukhatme (1985).

\section{Results and Discussion}

Part - 1: Effect of plant growth regulation techniques on branching habit

\section{Individual effect}

Individual effects of pinching and foliar application of PGRs were found significant on plant height, stem girth, number of leaves per plant, number of branches per plant and number of cuttings per plant for propagation of Euphorbia milii. Pinched plants $\left(\mathrm{P}_{1}\right)$ showed minimum plant height with thickest stems and maximum branches with higher number of leaves as compared to unpinched plants $\left(\mathrm{P}_{2}\right)$ during experiment (Table 1). Among PGRs application, $300 \mathrm{mg} / \mathrm{l}$ Alar $\left(\mathrm{R}_{6}\right)$ reduced plant height with thicker stems and increase number of branches per plant with maximum leaves in Euphorbia milii which was followed by or at par with application of $200 \mathrm{mg} / \mathrm{l}$ Alar $\left(\mathrm{R}_{5}\right)$ during experiment. 
Maximum number of cuttings was obtained from pinched plants as well as application of Alar at $300 \mathrm{mg} / \mathrm{l}$ concentration during both the seasons (Table 2).

\section{Interaction effect}

Foliar application of Alar at $300 \mathrm{mg} / \mathrm{l}$ in pinched plants $\left(\mathrm{P}_{1} \mathrm{R}_{6}\right)$ recorded minimum plant height at 30 DAS $(7.48$ and $7.30 \mathrm{~cm})$ as well as at 60 DAS (9.26 and $9.02 \mathrm{~cm}$ ) with thicker stems and higher number of leaves during first and second year, respectively.
Maximum plant height with minimum stem girth and leaves per plant was recorded in unpinched plants without any foliar application i.e. $\mathrm{P}_{2} \mathrm{R}_{10}$ at 30 and 60 DAS during experiment. Auxins are synthesized in shoot apical meristems and young leaves, promote elongation and cell enlargement as a result of an increase in the intensity of an osmotic driving force and tend to inhibit the activation for rapid elongation and division of stem tissue (Taiz and Zeiger, 2003) (Table 3$5)$.

Table.1 Effect of pinching and plant growth retardants on plant height of Euphorbia milii var.

'Pink Bold Beauty' at 30 and 60 DAS

\begin{tabular}{|c|c|c|c|c|c|c|c|c|c|c|c|c|}
\hline & \multicolumn{6}{|c|}{$2015-2016$} & \multicolumn{6}{|c|}{ 2016-2017 } \\
\hline & \multicolumn{3}{|c|}{30 DAS } & \multicolumn{3}{|c|}{ 60 DAS } & \multicolumn{3}{|c|}{30 DAS } & \multicolumn{3}{|c|}{60 DAS } \\
\hline & $\mathbf{P}_{1}$ & $\mathbf{P}_{2}$ & $\begin{array}{c}\text { Mean } \\
(\mathbf{R})\end{array}$ & $\mathbf{P}_{1}$ & $\mathbf{P}_{2}$ & Mean (R) & $\mathbf{P}_{1}$ & $\mathbf{P}_{2}$ & $\begin{array}{c}\text { Mean } \\
(\mathbf{R})\end{array}$ & $\mathbf{P}_{1}$ & $\mathbf{P}_{2}$ & $\begin{array}{c}\text { Mean } \\
\text { (R) }\end{array}$ \\
\hline $\mathbf{R}_{1}$ & 10.29 & 13.60 & 11.95 & 15.31 & 20.48 & 17.89 & 9.96 & 13.06 & 11.51 & 14.59 & 19.41 & 17.00 \\
\hline $\mathbf{R}_{2}$ & 9.44 & 12.88 & 11.16 & 13.49 & 18.92 & 16.20 & 9.08 & 12.45 & 10.76 & 12.87 & 18.04 & 15.45 \\
\hline $\mathbf{R}_{\mathbf{3}}$ & 9.17 & 12.52 & 10.84 & 12.89 & 18.14 & 15.52 & 8.84 & 12.04 & 10.44 & 12.33 & 17.28 & 14.80 \\
\hline $\mathbf{R}_{4}$ & 8.88 & 11.07 & 9.98 & 12.29 & 15.03 & 13.66 & 8.94 & 10.05 & 9.50 & 12.05 & 13.96 & 13.01 \\
\hline $\mathbf{R}_{5}$ & 7.76 & 10.71 & 9.24 & 9.87 & 14.25 & 12.06 & 7.57 & 10.10 & 8.83 & 9.58 & 13.53 & 11.56 \\
\hline $\mathbf{R}_{6}$ & 7.48 & 10.35 & 8.91 & 9.26 & 13.47 & 11.37 & 7.30 & 9.73 & 8.52 & 9.02 & 12.80 & 10.91 \\
\hline $\mathbf{R}_{7}$ & 12.52 & 16.49 & 14.51 & 20.14 & 26.69 & 23.42 & 11.69 & 16.65 & 14.17 & 18.65 & 25.96 & 22.31 \\
\hline Mean (P) & 9.37 & 12.52 & & 13.32 & 18.14 & & 9.05 & 12.01 & & 12.73 & 17.29 & \\
\hline & $\mathrm{P}$ & $\mathrm{R}$ & PXR & $\mathrm{P}$ & $\mathrm{R}$ & PXR & $\mathrm{P}$ & $\mathrm{R}$ & PXR & $\mathrm{P}$ & $\mathrm{R}$ & PXR \\
\hline S.Em. \pm & 0.05 & 0.09 & 0.13 & 0.02 & 0.04 & 0.06 & 0.22 & 0.42 & 0.59 & 0.31 & 0.59 & 0.83 \\
\hline C.D. & 0.14 & 0.26 & 0.37 & 0.06 & 0.12 & 0.17 & 0.65 & 1.20 & NS & 0.91 & 1.71 & NS \\
\hline
\end{tabular}

Table.2 Effect of pinching and plant growth retardants on stem girth of Euphorbia milii var. 'Pink Bold Beauty' at 30 and 60 DAS

\begin{tabular}{|c|c|c|c|c|c|c|c|c|c|c|c|c|}
\hline & \multicolumn{6}{|c|}{ 2015-2016 } & \multicolumn{6}{|c|}{ 2016-2017 } \\
\hline & \multicolumn{3}{|c|}{30 DAS } & \multicolumn{3}{|c|}{60 DAS } & \multicolumn{3}{|c|}{30 DAS } & \multicolumn{3}{|c|}{60 DAS } \\
\hline & $\mathbf{P}_{1}$ & $\mathbf{P}_{2}$ & $\begin{array}{c}\text { Mean } \\
\text { (R) }\end{array}$ & $\mathbf{P}_{1}$ & $\mathbf{P}_{2}$ & $\begin{array}{c}\text { Mean } \\
\text { (R) }\end{array}$ & $\mathbf{P}_{1}$ & $\mathbf{P}_{2}$ & $\begin{array}{c}\text { Mean } \\
\text { (R) }\end{array}$ & $\mathbf{P}_{1}$ & $\mathbf{P}_{2}$ & $\begin{array}{c}\text { Mean } \\
\text { (R) }\end{array}$ \\
\hline $\mathbf{R}_{1}$ & 2.02 & 1.50 & 1.76 & 2.24 & 1.72 & 1.98 & 1.91 & 1.35 & 1.63 & 2.45 & 1.81 & 2.13 \\
\hline $\mathbf{R}_{2}$ & 2.05 & 1.53 & 1.79 & 2.30 & 1.78 & 2.04 & 1.92 & 1.37 & 1.65 & 2.50 & 1.85 & 2.18 \\
\hline $\mathbf{R}_{\mathbf{3}}$ & 2.11 & 1.68 & 1.89 & 2.34 & 1.93 & 2.13 & 1.96 & 1.54 & 1.75 & 2.50 & 2.05 & 2.28 \\
\hline $\mathbf{R}_{4}$ & 2.21 & 1.79 & 2.00 & 2.45 & 2.01 & 2.23 & 2.04 & 1.65 & 1.85 & 2.63 & 2.16 & 2.39 \\
\hline $\mathbf{R}_{5}$ & 2.38 & 1.95 & 2.16 & 2.61 & 2.17 & 2.39 & 2.21 & 1.80 & 2.00 & 2.82 & 2.33 & 2.58 \\
\hline $\mathbf{R}_{6}$ & 2.44 & 2.02 & 2.23 & 2.68 & 2.26 & 2.47 & 2.26 & 1.87 & 2.07 & 2.88 & 2.44 & 2.66 \\
\hline $\mathbf{R}_{7}$ & 1.64 & 1.21 & 1.42 & 1.87 & 1.45 & 1.66 & 1.50 & 1.09 & 1.30 & 1.98 & 1.52 & 1.75 \\
\hline $\begin{array}{c}\text { Mean } \\
(\mathbf{P})\end{array}$ & 2.04 & 1.62 & & 2.36 & 1.90 & & 1.97 & 1.53 & & 2.54 & 2.02 & \\
\hline & $\mathrm{P}$ & $\mathrm{R}$ & PX R & $\mathrm{P}$ & $\mathrm{R}$ & PX R & $\mathrm{P}$ & $\mathrm{R}$ & PXR & $\mathrm{P}$ & $\mathrm{R}$ & PXR \\
\hline $\begin{array}{c}\text { S.Em. } \\
\pm\end{array}$ & 0.01 & 0.02 & 0.02 & 0.01 & 0.02 & 0.03 & 0.03 & 0.06 & 0.09 & 0.04 & 0.07 & 0.10 \\
\hline C.D. & 0.02 & 0.04 & NS & 0.03 & 0.05 & NS & 0.10 & 0.19 & NS & 0.11 & 0.20 & NS \\
\hline
\end{tabular}


Table.3 Effect of pinching and plant growth retardants on number of leaves per plant of Euphorbia milii var. 'Pink Bold Beauty' at 30 and 60 DAS

\begin{tabular}{|c|c|c|c|c|c|c|c|c|c|c|c|c|}
\hline & \multicolumn{6}{|c|}{ 2015-2016 } & \multicolumn{6}{|c|}{ 2016-2017 } \\
\hline & \multicolumn{3}{|c|}{30 DAS } & \multicolumn{3}{|c|}{60 DAS } & \multicolumn{3}{|c|}{30 DAS } & \multicolumn{3}{|c|}{60 DAS } \\
\hline & $\mathbf{P}_{1}$ & $\mathbf{P}_{2}$ & $\begin{array}{c}\text { Mean } \\
\text { (R) }\end{array}$ & $\mathbf{P}_{1}$ & $\mathbf{P}_{2}$ & Mean $(\mathbf{R})$ & $\mathbf{P}_{1}$ & $\mathbf{P}_{2}$ & $\begin{array}{c}\text { Mean } \\
\text { (R) }\end{array}$ & $\mathbf{P}_{1}$ & $\mathbf{P}_{2}$ & $\begin{array}{c}\text { Mean } \\
\text { (R) }\end{array}$ \\
\hline$R_{1}$ & 16.74 & 11.99 & 14.37 & 24.96 & 18.18 & 21.57 & 18.81 & 13.43 & 16.12 & 22.57 & 16.53 & 19.55 \\
\hline $\mathbf{R}_{2}$ & 19.28 & 13.27 & 16.27 & 27.53 & 19.47 & 23.50 & 21.56 & 14.91 & 18.24 & 24.56 & 17.52 & 21.04 \\
\hline $\mathbf{R}_{3}$ & 20.13 & 13.90 & 17.02 & 28.39 & 20.13 & 24.26 & 22.49 & 15.64 & 19.07 & 25.22 & 18.03 & 21.63 \\
\hline $\mathbf{R}_{4}$ & 20.97 & 16.46 & 18.72 & 29.24 & 22.72 & 25.98 & 23.62 & 18.42 & 21.02 & 25.89 & 20.03 & 22.96 \\
\hline $\mathbf{R}_{5}$ & 24.35 & 17.10 & 20.73 & 32.68 & 23.36 & 28.02 & 27.06 & 19.23 & 23.19 & 28.53 & 20.54 & 24.54 \\
\hline $\mathbf{R}_{6}$ & 25.20 & 17.73 & 21.47 & 33.54 & 24.01 & 28.77 & 27.96 & 20.07 & 24.02 & 29.20 & 21.03 & 25.12 \\
\hline $\mathbf{R}_{7}$ & 9.97 & 6.88 & 8.43 & 18.09 & 13.00 & 15.55 & 11.21 & 7.67 & 9.44 & 17.27 & 12.54 & 14.91 \\
\hline Mean (P) & 19.52 & 13.91 & & 27.78 & 20.12 & & 21.82 & 15.64 & & 24.75 & 18.03 & \\
\hline & $\mathrm{P}$ & $\mathrm{R}$ & P X R & $\mathrm{P}$ & $\mathrm{R}$ & P X R & $\mathrm{P}$ & $\mathrm{R}$ & P X R & $\mathrm{P}$ & $\mathrm{R}$ & PX R \\
\hline S.Em. \pm & 0.38 & 0.71 & 1.01 & 0.44 & 0.82 & 1.15 & 0.27 & 0.51 & 0.72 & 0.45 & 0.85 & 1.20 \\
\hline C.D. & 1.11 & 2.08 & NS & 1.27 & 2.37 & NS & 0.80 & 1.49 & NS & 1.32 & 2.47 & NS \\
\hline
\end{tabular}

Table.4 Effect of pinching and plant growth retardants on number of branches per plant of Euphorbia milii var. 'Pink Bold Beauty' at 30 and 60 DAS

\begin{tabular}{|c|c|c|c|c|c|c|c|c|c|c|c|c|}
\hline & \multicolumn{6}{|c|}{$2015-2016$} & \multicolumn{6}{|c|}{ 2016-2017 } \\
\hline & \multicolumn{3}{|c|}{30 DAS } & \multicolumn{3}{|c|}{60 DAS } & \multicolumn{3}{|c|}{30 DAS } & \multicolumn{3}{|c|}{60 DAS } \\
\hline & $\mathbf{P}_{1}$ & $\mathbf{P}_{2}$ & $\begin{array}{c}\text { Mean } \\
\text { (R) }\end{array}$ & $\mathbf{P}_{1}$ & $\mathbf{P}_{2}$ & $\begin{array}{c}\text { Mean } \\
\text { (R) }\end{array}$ & $\mathbf{P}_{1}$ & $\mathbf{P}_{2}$ & $\begin{array}{c}\text { Mean } \\
\text { (R) }\end{array}$ & $\mathbf{P}_{1}$ & $\mathbf{P}_{2}$ & $\begin{array}{c}\text { Mean } \\
\text { (R) }\end{array}$ \\
\hline $\mathbf{R}_{1}$ & 3.50 & 2.50 & 3.00 & 5.18 & 3.73 & 4.45 & 3.63 & 2.57 & 3.10 & 6.20 & 4.46 & 5.33 \\
\hline $\mathbf{R}_{2}$ & 4.20 & 2.86 & 3.53 & 6.06 & 4.18 & 5.12 & 4.28 & 3.00 & 3.64 & 7.28 & 5.03 & 6.16 \\
\hline $\mathbf{R}_{\mathbf{3}}$ & 4.44 & 3.04 & 3.74 & 6.35 & 4.41 & 5.38 & 4.50 & 3.21 & 3.85 & 7.65 & 5.32 & 6.48 \\
\hline $\mathbf{R}_{4}$ & 4.67 & 3.76 & 4.21 & 6.64 & 5.31 & 5.98 & 4.90 & 3.90 & 4.40 & 8.05 & 6.42 & 7.23 \\
\hline $\mathbf{R}_{5}$ & 5.61 & 3.93 & 4.77 & 7.82 & 5.54 & 6.68 & 5.64 & 4.21 & 4.93 & 9.44 & 6.74 & 8.09 \\
\hline $\mathbf{R}_{6}$ & 5.84 & 4.12 & 4.98 & 8.11 & 5.76 & 6.94 & 5.88 & 4.40 & 5.15 & 9.80 & 7.03 & 8.42 \\
\hline $\mathbf{R}_{7}$ & 1.62 & 1.06 & 1.34 & 2.81 & 1.94 & 2.38 & 1.66 & 1.05 & 1.35 & 3.26 & 2.20 & 2.73 \\
\hline $\begin{array}{c}\text { Mean } \\
\text { (P) }\end{array}$ & 4.27 & 3.04 & & 6.14 & 4.41 & & 4.36 & 3.19 & & 7.38 & 5.32 & \\
\hline & $\mathrm{P}$ & $\mathrm{R}$ & P X R & $\mathrm{P}$ & $\mathrm{R}$ & P X R & $\mathrm{P}$ & $\mathrm{R}$ & PX R & $\mathrm{P}$ & $\mathrm{R}$ & PX R \\
\hline $\begin{array}{c}\text { S.Em. } \\
\pm\end{array}$ & 0.04 & 0.07 & 0.10 & 0.08 & 0.14 & 0.20 & 0.05 & 0.09 & 0.13 & 0.10 & 0.18 & 0.26 \\
\hline C.D. & 0.11 & 0.20 & 0.27 & 0.22 & 0.42 & 0.59 & 0.14 & 0.27 & 0.39 & 0.28 & 0.53 & 0.75 \\
\hline
\end{tabular}

Table.5 Effect of pinching and plant growth retardants on number of cuttings per plant of Euphorbia milii var. 'Pink Bold Beauty'

\begin{tabular}{|c|c|c|c|c|c|c|}
\hline & \multicolumn{3}{|c|}{$\mathbf{2 0 1 5 - 2 0 1 6}$} & \multicolumn{3}{c|}{$\mathbf{2 0 1 6 - 2 0 1 7}$} \\
\cline { 2 - 7 } & $\mathbf{P}_{\mathbf{1}}$ & $\mathbf{P}_{\mathbf{2}}$ & Mean (R) & $\mathbf{P}_{\mathbf{1}}$ & $\mathbf{P}_{\mathbf{2}}$ & Mean (R) \\
\hline $\mathbf{R}_{\mathbf{1}}$ & 4.47 & 3.26 & 3.87 & 4.71 & 3.43 & 4.07 \\
\hline $\mathbf{R}_{\mathbf{2}}$ & 5.28 & 3.61 & 4.45 & 5.54 & 3.80 & 4.67 \\
\hline $\mathbf{R}_{\mathbf{3}}$ & 5.55 & 3.79 & 4.67 & 5.80 & 3.99 & 4.90 \\
\hline $\mathbf{R}_{\mathbf{4}}$ & 5.70 & 4.62 & 5.16 & 5.97 & 4.82 & 5.40 \\
\hline $\mathbf{R}_{\mathbf{5}}$ & 6.91 & 4.69 & 5.80 & 7.18 & 4.92 & 6.05 \\
\hline $\mathbf{R}_{\mathbf{6}}$ & 7.19 & 4.88 & 6.03 & 7.47 & 5.10 & 6.29 \\
\hline $\mathbf{R}_{\mathbf{7}}$ & 2.47 & 1.73 & 2.10 & 2.66 & 1.86 & 2.26 \\
\hline Mean (P) & 5.37 & 3.80 & & 5.62 & 3.99 & \\
\hline & $\mathrm{P}$ & $\mathrm{R}$ & $\mathrm{P}$ X R & $\mathrm{P}$ & $\mathrm{R}$ & $\mathrm{P}$ X R \\
\hline S.Em. \pm & 0.09 & 0.17 & 0.24 & 0.09 & 0.18 & 0.25 \\
\hline C.D. & 0.27 & 0.50 & 0.71 & 0.27 & 0.51 & 0.72 \\
\hline
\end{tabular}


Table.6 Effect of different rooting media and IBA concentrations on shoot parameters of Euphorbia milii cutting var. 'Pink Bold Beauty'

\begin{tabular}{|c|c|c|c|c|c|c|c|c|c|c|c|c|c|c|c|c|c|c|c|c|c|c|c|c|}
\hline & \multicolumn{8}{|c|}{ Number of days to new sprout } & \multicolumn{8}{|c|}{ Percentage of Sprouted Cuttings } & \multicolumn{8}{|c|}{ Length of Shoot (cm) } \\
\hline & \multicolumn{4}{|c|}{ 2015-2016 } & \multicolumn{4}{|c|}{ 2016-17 } & \multicolumn{4}{|c|}{ 2015-2016 } & \multicolumn{4}{|c|}{ 2016-17 } & \multicolumn{5}{|c|}{ 2015-2016 } & \multicolumn{3}{|c|}{ 2016-17 } \\
\hline & $\mathbf{M}_{1}$ & $\mathbf{M}_{2}$ & $\mathbf{M}_{3}$ & $\begin{array}{c}\text { Mean } \\
\text { (I) }\end{array}$ & $\mathbf{M}_{1}$ & $\mathbf{M}_{2}$ & $\mathbf{M}_{3}$ & $\begin{array}{c}\text { Mean } \\
\text { (I) }\end{array}$ & $\mathbf{M}_{1}$ & $\mathbf{M}_{2}$ & $\mathbf{M}_{3}$ & $\begin{array}{c}\text { Mean } \\
\text { (I) }\end{array}$ & $\mathbf{M}_{1}$ & $\mathbf{M}_{2}$ & $\mathbf{M}_{3}$ & $\begin{array}{c}\text { Mean } \\
\text { (I) }\end{array}$ & $\mathbf{M}_{1}$ & $\mathbf{M}_{2}$ & $\mathbf{M}_{3}$ & $\begin{array}{c}\text { Mean } \\
\text { (I) }\end{array}$ & $\mathbf{M}_{1}$ & $\mathbf{M}_{2}$ & $\mathbf{M}_{3}$ & $\begin{array}{c}\text { Mean } \\
\text { (I) }\end{array}$ \\
\hline$I_{1}$ & 32.01 & 31.44 & 36.93 & 33.46 & 31.45 & 29.86 & 34.58 & 31.97 & 77.45 & 80.84 & 68.29 & 75.53 & 72.36 & 73.82 & 71.28 & 72.48 & 10.92 & 11.11 & 10.80 & 10.94 & 10.72 & 10.81 & 10.35 & 10.63 \\
\hline $\mathbf{I}_{2}$ & 28.84 & 28.17 & 37.59 & 31.53 & 26.94 & 29.58 & 32.77 & 29.76 & 84.96 & 88.80 & 82.57 & 85.44 & 80.17 & 86.10 & 76.21 & 80.83 & 11.73 & 12.46 & 11.35 & 11.84 & 11.93 & 12.55 & 11.54 & 12.01 \\
\hline $\mathbf{I}_{3}$ & 30.41 & 26.16 & 32.20 & 29.59 & 31.00 & 27.13 & 32.94 & 30.35 & 85.00 & 90.21 & 83.20 & 86.14 & 82.69 & 83.03 & 77.64 & 81.12 & 11.92 & 12.77 & 11.61 & 12.10 & 12.12 & 12.59 & 11.60 & 12.10 \\
\hline$I_{4}$ & 28.29 & 25.99 & 31.11 & 28.46 & 25.47 & 24.06 & 30.22 & 26.58 & 87.16 & 94.19 & 84.66 & 88.67 & 82.80 & 87.11 & 77.83 & 82.58 & 12.20 & 13.18 & 11.71 & 12.38 & 12.44 & 12.75 & 11.72 & 12.30 \\
\hline \multirow[t]{2}{*}{$\begin{array}{c}\text { Mean } \\
(\mathbf{M})\end{array}$} & 29.89 & 27.94 & 34.46 & & 28.71 & 27.66 & 32.62 & & 83.64 & 88.51 & 79.68 & & 79.51 & \begin{tabular}{|l|}
83.77 \\
\end{tabular} & 75.74 & & 11.69 & 12.38 & 11.38 & & 11.80 & 12.18 & 11.30 & \\
\hline & $\mathrm{M}$ & $\mathrm{I}$ & \multicolumn{2}{|c|}{ MXI } & $\mathrm{M}$ & $\mathrm{I}$ & $\mathrm{M}$ & X I & $\mathrm{M}$ & $\mathrm{I}$ & \multicolumn{2}{|c|}{ MXI } & $\mathrm{M}$ & I & & X I & $\mathrm{M}$ & $\mathrm{I}$ & \multicolumn{2}{|c|}{ MX I } & $\mathrm{M}$ & $\mathrm{I}$ & \multicolumn{2}{|c|}{ M X I } \\
\hline $\begin{array}{c}\text { S.Em. } \\
\pm\end{array}$ & 0.70 & 0.81 & \multicolumn{2}{|c|}{1.40} & 0.70 & 0.80 & 1. & 39 & 2.36 & 2.72 & \multicolumn{2}{|c|}{4.71} & 2.43 & 2.81 & & 86 & 0.28 & 0.33 & \multicolumn{2}{|c|}{0.57} & 0.33 & 0.38 & \multicolumn{2}{|c|}{0.66} \\
\hline C.D. & 2.05 & 2.36 & \multicolumn{2}{|c|}{ NS } & 2.04 & 2.36 & $\mathrm{~N}$ & IS & 6.92 & 7.99 & \multicolumn{2}{|c|}{$\mathrm{NS}$} & NS & 8.24 & & $\mathrm{NS}$ & NS & 0.92 & \multicolumn{2}{|c|}{ NS } & NS & 1.12 & \multicolumn{2}{|c|}{ NS } \\
\hline
\end{tabular}

Table.7 Effect of different rooting media and IBA concentrations on root parameters of Euphorbia milii cutting var. 'Pink Bold Beauty'

\begin{tabular}{|c|c|c|c|c|c|c|c|c|c|c|c|c|c|c|c|c|c|c|c|c|c|c|c|c|}
\hline & \multicolumn{8}{|c|}{ Number of roots per cutting } & \multicolumn{8}{|c|}{ Length of roots (cm) } & \multicolumn{8}{|c|}{ Survival Percentage } \\
\hline & \multicolumn{4}{|c|}{$2015-2016$} & \multicolumn{4}{|c|}{ 2016-17 } & \multicolumn{4}{|c|}{$2015-2016$} & \multicolumn{4}{|c|}{ 2016-17 } & \multicolumn{4}{|c|}{$2015-2016$} & \multicolumn{4}{|c|}{ 2016-17 } \\
\hline & $\mathbf{M}_{1}$ & $\mathbf{M}_{2}$ & $\mathbf{M}_{3}$ & \begin{tabular}{|c} 
Mean \\
(I)
\end{tabular} & $\mathbf{M}_{1}$ & $\mathbf{M}_{2}$ & $\mathbf{M}_{3}$ & \begin{tabular}{|c}
$\begin{array}{c}\text { Mean } \\
\text { (I) }\end{array}$ \\
\end{tabular} & $\mathbf{M}_{1}$ & $\mathbf{M}_{2}$ & $\mathbf{M}_{3}$ & $\begin{array}{c}\text { Mean } \\
\text { (I) }\end{array}$ & $\mathbf{M}_{1}$ & $\mathbf{M}_{2}$ & $\mathbf{M}_{3}$ & $\begin{array}{c}\text { Mean } \\
\text { (I) }\end{array}$ & $\mathbf{M}_{1}$ & $\mathbf{M}_{2}$ & $\mathbf{M}_{3}$ & \begin{tabular}{|c}
$\begin{array}{c}\text { Mean } \\
\text { (I) }\end{array}$ \\
\end{tabular} & $\mathbf{M}_{1}$ & $\mathbf{M}_{2}$ & $\mathbf{M}_{3}$ & $\begin{array}{c}\text { Mean } \\
\text { (I) }\end{array}$ \\
\hline$I_{1}$ & 10.10 & 10.37 & 9.14 & 9.87 & 9.68 & 9.77 & 9.51 & 9.65 & 5.12 & 5.21 & 4.87 & 5.07 & 4.92 & 5.30 & 4.91 & 5.05 & 65.95 & 69.68 & 67.83 & 67.82 & 76.12 & 63.56 & 59.68 & 66.45 \\
\hline$I_{2}$ & 12.71 & 12.90 & 11.24 & 12.28 & 12.09 & 13.08 & 10.72 & \begin{tabular}{|l|}
11.96 \\
\end{tabular} & 5.68 & 6.55 & 5.34 & 5.86 & 6.15 & 7.27 & 5.42 & 6.28 & 72.82 & 80.20 & 72.92 & 75.32 & 71.72 & 73.48 & 73.23 & 72.81 \\
\hline $\mathbf{I}_{3}$ & 12.79 & 13.09 & 11.49 & 12.46 & 12.10 & 13.86 & 10.86 & 12.27 & 6.34 & 7.68 & 5.35 & 6.46 & 6.72 & 7.74 & 5.57 & 6.68 & 74.99 & 76.07 & 69.55 & 73.53 & 65.16 & 84.42 & 68.06 & 74.08 \\
\hline $\mathbf{I}_{4}$ & 12.82 & 14.37 & 12.19 & 13.13 & 12.40 & 15.71 & 11.84 & 13.32 & 6.43 & 7.98 & 5.57 & 6.66 & 7.20 & 9.27 & 5.69 & 7.39 & 78.14 & 91.52 & 68.11 & 79.26 & 69.00 & 89.03 & 69.69 & 75.91 \\
\hline \multirow[t]{2}{*}{$\begin{array}{c}\text { Mean } \\
\text { (M) }\end{array}$} & 12.11 & 12.68 & 11.02 & & 11.57 & 13.10 & 10.73 & & 5.89 & 6.86 & 5.28 & & 6.25 & 7.40 & 5.40 & & 72.98 & 79.36 & 69.90 & & 70.50 & 77.62 & 67.67 & \\
\hline & $\mathrm{M}$ & I & \multicolumn{2}{|c|}{ MXI } & $\mathrm{M}$ & I & \multicolumn{2}{|c|}{ MXI } & M & 1 & \multicolumn{2}{|c|}{ M X I } & $\mathrm{M}$ & $\mathrm{I}$ & & IXI & $\mathrm{M}$ & I & \multicolumn{2}{|c|}{ MXI } & $\mathrm{M}$ & I & \multicolumn{2}{|c|}{ M X I } \\
\hline S.Em. \pm & 0.31 & 0.36 & \multicolumn{2}{|c|}{0.62} & 0.33 & 0.38 & \multicolumn{2}{|c|}{0.66} & 0.12 & 0.14 & \multicolumn{2}{|c|}{0.23} & 0.13 & 0.15 & & 0.26 & 1.58 & 1.83 & \multicolumn{2}{|c|}{3.16} & 1.95 & 2.26 & \multicolumn{2}{|c|}{3.91} \\
\hline C.D. & 0.91 & 1.05 & \multicolumn{2}{|c|}{ NS } & 0.97 & 1.12 & $\mathrm{~N}$ & IS & 0.34 & 0.40 & \multicolumn{2}{|c|}{0.69} & 0.38 & 0.44 & & 0.76 & 4.64 & 5.36 & \multicolumn{2}{|c|}{9.28} & 5.74 & NS & \multicolumn{2}{|c|}{11.48} \\
\hline
\end{tabular}


Reduction of the shoot growth and internodal length of many ornamental plants can be obtained by the use of synthetic plant growth retardants like Alar and TIBA (Warner and Erwin, 2003) and by elimination of the apical dominance (Lodeta et al., 2010 and Hashemabadi et al., 2012). According to Menhennet (1980), Alar blocks particularly $3 \beta$-hydroxylation, which inhibits formation of highly active $\mathrm{GA}_{3}$ from inactive precursors at late stage (stage 3) of GA metabolism and thereby affects plant height in plants. The application of Alar has been shown to reduce the plant height in a number of plant species such as Chrysanthemum (Kahar, 2008) and Poinsettia (Lodeta et al., 2010). Increase in production of more leaves may be related to increase in number of branches due to inhibition of apical dominance caused by auxin and diversion of photosynthets towards the axillary buds as explained earlier in China aster by Gyandev (2006) and in rose by Shivangi (2012).

Pinched plants treated with Alar at $300 \mathrm{mg} / \mathrm{l}$ $\left(\mathrm{P}_{1} \mathrm{R}_{6}\right)$ significantly produced maximum number of branches at 30 DAS (5.84 and 5.88) and at 60 DAS (8.11 and 9.80) as well as maximum number of cuttings (7.19 and 7.47) which was at par with $\mathrm{P}_{1} \mathrm{R}_{8}$ treatment combination. Minimum number of branches and number of cuttings were observed in unpinhced plants without any retardants treatment i.e. $\mathrm{P}_{2} \mathrm{R}_{10}$ during both the seasons. Vegetative or generative organs in ornamental plant production can be manipulated by removing of apical buds for controlling growth parameters and to increase branching production. Heuvelink and Buiskool (1995) and Jovicich et al., (1999) postulated that after removal of apical bud the sink-source ratio is influenced which alters the growth parameters and enhances branching pattern in plants. Influence of pinching on vegetative growth has been similar in carnation (Dalal et al., 2006) and chrysanthemum (Singh and Baboo, 2003). Further, suppression of apical dominance induces the lateral vegetative bud growth (Cathey and Stuart, 1961) which leads to increase number of branches and ultimately in the plant spread.

Part - 2: Effect of IBA and media on rooting of semi hardwood cuttings of Euphorbia milii

Cuttings stumped in $\mathrm{M}_{2}$ media i.e. cocopeat showed highest percentage of sprouted cuttings (88.51 and $83.77 \%$ ) with longest shoots $(12.38$ and $12.18 \mathrm{~cm})$ and early sprouting (27.94 and 27.66 days) which was followed by sand media i.e. $\mathrm{M}_{1}$ during first and second year respectively. Further, treatment of $750 \mathrm{mg} / \mathrm{l}$ IBA $\left(\mathrm{I}_{4}\right)$ showed maximum sprouting percentage $(88.67$ and $82.58 \%)$ with longest shoots $(12.38 \mathrm{~cm}$ and $12.18 \mathrm{~cm}$ ) which took minimum days for new sprouts (28.46 and 26.58 days) which was statistically at par with $500 \mathrm{mg} / \mathrm{l}\left(\mathrm{I}_{3}\right)$ and 250 $\mathrm{mg} / \mathrm{l}$ IBA $\left(\mathrm{I}_{2}\right)$ concentration during both the years. Lowest percentage of sprouted cutting with delay in sprouting was noted in untreated cuttings $\left(\mathrm{I}_{1}\right)$ during experiment. However, interaction effect of rooting media and IBA concentration was found non-significant. Minimum days for sprouting with highest percentage of sprouted cuttings was a result of good drainage in cocopeat media as well as in sand media, which kept media free from microbial action in periphery of rooting region of cuttings. An appropriate rooting medium generally has to have an optimal volume of gas filled pore space and oxygen diffusion rate adequate for the needs of respiration (Fonteno and Nelson, 1990). Maximum growth of cutting and biomass was probably due to the suitable combination of physical condition comprising of water holding condition and porosity of the cocopeat and sand media. Application of IBA at different concentrations had significant effect on shoot parameters. The stored food material present in the cutting is mobilized with the aid of growth regulators, which 
hastens sprouting. Early sprouting of shoots with more number of leaves and shoots with auxin treatments was also observed in bougainvillea (Seyedi et al., 2014) and in pink mussanda (Patel, 2009).

Cuttings grown in cocopeat media $\left(\mathrm{M}_{2}\right)$ showed significantly higher number of roots (12.68 and 13.10) and longest roots (6.86 and $7.40 \mathrm{~cm}$ ) during first and second year, respectively. Whereas minimum number of shortest roots were noted in the cuttings grown in media $\mathrm{M}_{3}$ i.e. mixture of cocopeat and banana pseudostem fibers during the experiment. In case of IBA application, cuttings treated with $750 \mathrm{mg} / \mathrm{l}$ IBA recorded maximum number of roots (13.13 and 13.32) and longest roots $(6.66$ and $7.39 \mathrm{~cm})$ during both the seasons, respectively. Untreated cuttings $\left(\mathrm{I}_{1}\right)$ exhibited minimum number of roots per cuttings and root length during the experiment. In addition, treatment combination $\mathrm{M}_{2} \mathrm{I}_{4}$ i.e. application of $750 \mathrm{mg} / \mathrm{l}$ IBA with cocopeat media resulted in longest root per cutting during first year $(7.98 \mathrm{~cm})$ and second year $(9.27 \mathrm{~cm})$ which was significantly at par with $\mathrm{M}_{2} \mathrm{I}_{3}$ treatment combination. Ofori-Gyamfi (1998) reported that rooting performance depends on the type of medium and its physical properties used in the propagating structure. The beneficial effect of root formation in stem-cuttings may be affected by physical and chemical characteristics of rooting substrates viz. media texture, structure, bulk density, porosity, water-holding capacity, $\mathrm{pH}$, temperature and oxygen etc. (Kester et al., 1990 and Larsen and Guse, 1997) which allows promotion or inhibition of root growth (Hartmann et al., 1997). The differences in rooting ability of various species propagated in different rooting media could be explained by xeromorphic or hydromorphic status of media (Loach, 1992) and the effects of this status on the water relations of the cuttings (Mensen et al., 1997). Improvement of the formation and growth of roots of Euphorbia cuttings in cocopeat subtract could be linked to a likely increase of air content and oxygen diffusion rate than in sand media as also reported by Gislerod (1983) for Poinsettia cuttings.

Individual effect of rooting media and IBA concentrations significantly influenced survival rate of Euphorbia milii cuttings during both the seasons. In case of combined effect of rooting media and IBA concentrations, maximum survival percentage was recorded in (91.52 and $89.03 \%)$ in the cuttings grown in cocopeat media with treatment of $750 \mathrm{mg} / \mathrm{l} \mathrm{IBA}\left(\mathrm{M}_{2} \mathrm{I}_{4}\right)$ which was followed by $\mathrm{M}_{2} \mathrm{I}_{3}$ treatment combination during first and second year. However, minimum survival percentage recorded in untreated cuttings grown in mixture of cocopeat + banana pseudostem fiber i.e. $\mathrm{M}_{3} \mathrm{I}_{1}$ (Table 6 and 7). According to Davis and Hassig (1990), the production of adventitious roots in plants through cell division, multiplication and specialization is also controlled by plant growth substances especially auxins. Auxin has been well known to promote adventitious root formation and accelerate root initiation (Lee et al., 2000; Ibàñez-Torres, 2004) and increase the number and percentage of rooted cuttings as well as quality of roots produced per cutting (Leonardi et al., 2001). Enhanced rooting of the Euphorbia stem cuttings treated with 750 $\mathrm{mg} / \mathrm{l}$ IBA concentration is concordant with the findings by Aminah et al., (2006) in which application of $0.8 \%$ IBA recorded the highest rooting percentage in stem cuttings of Shorea parvifolia and Shorea macroptera. Further, better root initiation and root growth lead to higher survival percentage with decrease in mortality rate by rotting of Euphorbia cuttings as also reported by Jo (1990). Auxins at different concentrations have been reported to differentially influence adventitious rooting and establishment rate in Poinsettia pulcherrima (Shirol et al., 1992 and Sochacki and Chmiel, 1994) and in Bougainvillea (Sahariya et al., (2013). 


\section{References}

Aminah H, Nor Hasnita RMN and Hamzah M. 2006. Effects of Indolebutyric acid concentrations and media on rooting of leafy cuttings of Shorea parvifolia and Shorea macroptera. J. Trop. For. Sci., 18(1): 1-7.

Cathey MH and Stuart NW. 1961. Comparative plant growth retarding activity of AMO1618, phosphorus and CCC. Bot. Gaz., 123: 51-57.

Dalal SR, Nandre DR, Bharad SG, Swarupa U and Shinde RD. 2006. Effect of pinching on carnation cv. Yellow Solar under polyhouse condition. Int. J. Agri. Sci., 2(2): 356-357.

Davis DT and Hassig BE. 1990. Chemical control of adventitious root formation in cuttings. Bull. Plant Growth Regul. Soc. Am., 18: 117.

Fonteno WC and Nelson PV. 1990. Physical properties of plant responses to rockwool amended media. J. Am. Soc. Hortic. Sci., 115(3): 375-381.

Gislerod, HR. 1983. Physical conditions of propagation media and their influence on the rooting of cuttings III. The effect of air content and temperature in different propagation media on the rooting of cuttings. Plant Soil, 75: 1-14.

Gyandev B. 2006. Effect of pinching, plant nutrition and growth retardants on seed yield, quality and storage studies China aster [Callistephus chinensis (L.) Nees.]. M.Sc. Thesis submitted to University of Agricultural Sciences, Dharwad, Karnataka.

Hartmann HT, Kester DE, Davies FT and Geneve RL. 1997. Plant propagation: principles and practices. $7^{\text {th }}$ Ed. Prentice-Hall, Englewood Cliffs, N.J., USA, 880.

Hashemabadi D, Lipaei RS, Shadparvar V, Zarchini M and Kaviani B. 2012. The effect of cycocel and daminozide on some growth and flowering characteristics of Calendula officinalis L., an ornamental and medicinal plant. J. Med. Plants Res., 6(9): 1752-1757.

Heuvelink E and Buiskool RPM. 1995. Influence of sink-source interaction on dry matter production in tomato. Ann. Bot., 75: 381389.

Ibàñez-Torres A. 2004. Rooting experiments with
Euphorbia lagascae cuttings. Anales de Biologia, 26: 101-104.

Jankalski S. 2000. Crown of Thorns hybrids- Past and present. Cactus Succulent J., 72: 202204.

Jo IS. 1995. Effect of organic fertilizer on soil physical properties and plant growth. In: The use of organic fertilizer in crop production, Suwon, South Korea, 25.

Jovicich E, Cantliffe DJ and Hochmuth GJ. 1999. Plant density and shoot pruning on fruit yield and quality of a summer greenhouse sweet pepper crop in north central Florida. In: K.D. Batal (ed.). $28^{\text {th }}$ Natl. Agr. Plastics Congr. Proc. Amer. Soc. Plasticulture, Tallahassee, American Society for Plasticulture, State College, PA. 184-190.

Kahar SAb. 2008. Effect of frequency and concentration of B-9 (Daminozide) on growth, flowering and flower quality of 'Reagan Sunny' Chrysanthemum (Chrysanthemum morifolium Ramat.). Acta Horticulturae, 788:141-145.

Kester DE, Hartmann TH and Davier FT. 1990. Plant propagation: Principles and Practices. $5^{\text {th }}$ edn, Prentice Hall, Singapore, 647.

Larsen FE and Guse WE. 1997. Propagating deciduous and evergreen shrubs, trees and vines with stem cuttings. A Pacific Northwest Cooperative Extension Publication, Washington, USA, 10.

Lee AK, Suh JK and Roh MS. 2000. Propagation of Ardisia species native to Korea by seeds or by rooting of stem tip cuttings. Acta Horticulturae, 541: 135-145.

Lee JH, Jine ES and Kim WT. 1999. Inhibition of auxine-induced ethylene production by SA in mungbean hypocotyls. J. Plant Bid., 42: 1-7.

Leonardi C, Ruggeri A and Malta S. 2001. Hormone effects on in vitro proliferation and rooting of Grevillea explants. Sci. Hortic., 90: 335-341.

Loach K. 1992. Environmental conditions for rooting cuttings: Importance, measurement and control. Acta Horticulturae, 314: 233242.

Lodeta KB, Ban SG, Percia S, Dumicic G and Bucan L. 2010. Response of poinsettia to drench application of growth regulators. $J$. food Agric. Environ., 8(1): 297-301. 
Menhennet R. 1980. Evidence that daminozide, but not two other growth retardants, modifies the fate of applied gibberellins $\mathrm{A}_{9}$ in Chrysanthemum morifolium Ramat. $J$. Ext. Bot., 31: 1631-1642.

Mensen F, Newton, AC and Leakey RRB. 1997. Vegetative propagation of Cordia alliodora (Ruiz \& Pavon) Oken: The effects of IBA concentration, propagation medium and cutting origin. For. Ecol. Manage., 92: 4554.

Ofori-Gyamfi E. 1998. Investigation in some factors affecting vegetative propagation of Coffee (Coffee conaephera var. Robusta Pierve). MPhil. Thesis submitted to University of Cape Coast, Ghana.

Panse VG and Sukhamte PV. 1985. Statistical Methods for Agricultural Workers. ICAR Pub., New Delhi.

Patel, PC. 2009. Bio efficacy of plant growth regulator and bio stimulant on propagation of Mussanda Pink. M.Sc. Thesis submitted to Navsari Agricultural University, Navsari.

Sahariya K, Singh JN and Singh A. 2013. Studies on the effect of IBA on rooting of bougainvillea var. Thimma cuttings in open field and polyhouse conditions. Asian J. Hort., 8(1):140-142.

Seyedi A, Esmaeili A, Zadesh K and Porsiabidi M. 2014. Evaluation of Propagation of Bougainvillea (Bougainvillea glabera. L) through Stenting Method in Response to Different IBA Concentrations and Rootstocks. IJFAS J., 3(8): 72-875.

Shirol AM, Patil AA and Nalwadi UG. 1992. Biochemical basis of Euphorbia pulcherrima Willd. Var. Alba. Hort. Rooting through cuttings. South Indian Hort., 40: 159-165.

Shivangi Seth. 2012. Pot culture management in rose cv. Valentine with the use of plant growth retardants under polyhouse condition. M.Sc. Thesis submitted to Navsari Agricultural University, Navsari.

Singh MK and Baboo R. 2003. Response of nitrogen, potassium and pinching levels on growth and flowering in chrysanthemum. $J$. Ornm. Hort., 6(4): 390-393.

Smoley R. 2000. Giant-flowered Euphorbia milii hybrids. Cactus Succulent J., 72:198-201.

Sochacki D and Chmiel H. 1994. Effect of several factors on rooting of poinsettia (Euphorbia pulcherrima Willd.). Zeszyty Naukowe Instytutu Sadownictwa-i- Kwiaciarstwa-wSkierniewieach, 1: 77-84.

Taiz L and Zeiger E. 2003. Growth and development. In: Plant Physiology, Edn $3^{\text {rd }}$ Parima publishing corporation, New Delhi, India, 309-591.

Warner RM and Ervin JE. 2003. Effect of plant growth retardants on stem elongation of hibiscus species. Hort. Tech., 13: 293-296.

\section{How to cite this article:}

Kapadiya, D.B., Alka Singh, A.J. Bhandari, A.I. Patel and Krishna Patel. 2017. Development of in vivo Plant Propagation Protocol in Euphorbia milii var. 'Pink Bold Beauty'. Int.J.Curr.Microbiol.App.Sci. 6(12): 141-149. doi: https://doi.org/10.20546/ijcmas.2017.612.019 